\title{
EXPLOTACIÓN SOSTENIBLE DE RECURSOS NATURALES EN ZONAS ESPECIALES DE CONSERVACIÓN (ZEC) QUE CONFORMAN LA RED NATURA 2000 DE LA UNIÓN EUROPEA: UN ANÁLISIS DE LOS PROYECTOS MINEROS
}

\author{
GABRIEL BALLESTEROS PINILLA ${ }^{1}$ \\ gabriel.ballesteros@ustabuca.edu.co
}

CRISTINA MONTES CHIVITE ${ }^{2}$

cristina@monteschivite.com

\section{Resumen}

El presente trabajo presenta una breve reflexión sobre el concepto integridad de la ZEC, como elemento sustancial dentro del procedimiento de evaluación adecuada que debe seguir todo plan o proyecto. Este es, en definitiva, el último filtro por el que deberán pasar los promotores de actividades en zonas protegidas. Para definir la integridad de la ZEC deberá tenerse en cuenta el contexto en el que se desarrollan los proyectos, la norma de su creación y la directiva hábitats, de acuerdo con los criterios doctrinales y jurisprudenciales que lo han interpretado, así como con las conside-

1 Profesor en la Universidad Santo Tomás, Seccional Bucaramanga y Universidad del País Vasco.

2 Abogada especializada en derecho administrativo. Máster en Derecho Medioambiental, Universidad del País Vasco. 
raciones y análisis provenientes de la propia Comisión Europea, haciendo especial referencia a los intereses en juego del sector minero, como se muestra en este art.. Finalmente, se exponen algunas prácticas de minería sostenible que podrían implementarse en las ZECs, permitiendo un desarrollo socioeconómico compatible con la protección del medio ambiente.

\title{
Palabras clave
}

Biodiversidad; directiva hábitats; integridad de la ZEC; ZEC; CDB; evaluación adecuada; objetivos de conservación; Red Natura 2000; coherencia; minería sostenible

\section{SUSTAINABLE USE OF NATURAL RESOURCES IN SPECIAL AREAS OF CONSERVATION (SACS) OF THE EUROPEAN UNION NATURA 2000 NETWORK: AN ANALYSIS OF MINING PROJECTS}

\begin{abstract}
This paper presents a brief reflection on the concept of integrity of the Special Conservation Areas (SCA), as a substantial element in the proper evaluation procedure, which should follow any plan or project. This is the last filter by which promoters of activities in protected areas must pass. In order to define the integrity of the SAC, account must be taken of the context in which the projects are developed, the way in which they are established and the Habitats Directive, in accordance with the doctrinal and jurisprudential criteria which have interpreted it, as well as the considerations and analysis from the European Commission itself, with special reference to the interests at stake of the mining sector, as shown in this article. Finally, some sustainable mining practices that could be implemented in the SACs are presented, allowing socio-economic development compatible with the protection of the environment.
\end{abstract}

\section{Keywords}

Biodiversity; habitats directive; integrity of ZEC; ZEC; CBD; appropriate assessment; conservation objectives; Red Natura 2000; coherence; sustainable mining

\section{L'EXPLOITATION SOUTENABLE DES RESSOURCES NATURELLES DANS LES ZONES SPECIALES DE CONSERVATION (ZSC) DU RESEAU NATURA 2000 DE L'UNION EUROPEENNE: UNE ANALYSE DES PROJETS MINIERS}

\section{Résumé}

Cet travail présente une brève réflexion sur le concept de l'intégrité de la ZSC, comme un élément substantiel dans la procédure d'une évaluation adéqua- 
te qui doit suivre tout plan ou projet et, en définitive, comme un dernier filtre qui devra être justifié par les promoteurs de toute activité dans des zones protégées pour l'implantation de tels plans ou projets, toujours relié depuis le contexte où se situe dans la norme de sa création, la Directive habitats, et avec les critères doctrinaux et jurisprudentiels qui l'ont interprétée, ainsi qu'avec les considérations et les analyses provenant de la Commission européenne elle-même, en faisant référence spéciale aux intérêts en jeu du secteur minier. Finalement, on montre quelques exemples d'industrie minière soutenable qui pourraient être développés dans les ZSCs, en permettant un développement socio-économique compatible avec la protection de l'environnement.

\section{Mots cles}

Biodiversité; directive habitats; intégrité de la ZSC; ZSC; CDB; évaluation appropriée; objectifs de conservation; Red Natura 2000; cohérence; exploitation minière durable. 


\section{SUMARIO}

I. INTRODUCCIÓN. II. DIVERSIDAD BIOLÓGICA Y SU PROTECCIÓN: EL CONCEPTO DE INTEGRIDAD DE LAS ÁREAS PROTEGIDAS. 1. La Protección de la Diversidad Biológica en el Derecho Internacional. 2. La directiva hábitats. III. ANÁLISIS JURISPRUDENCIAL DEL CONCEPTO DE INTEGRIDAD DE LA ZEC IV. APLICACIÓN DEL ANÁLISIS AL SECTOR MINERO. V. BUENAS PRÁCTICAS PARA UNA MINERÍA SOSTENIBLE. 1. La protección ambiental en la minería sostenible. 2. El factor económico en la minería sostenible. 3. El factor social en la minería sostenible. VI. CONCLUSIONES.

\section{INTRODUCCIÓN}

Para compatibilizar intereses muchas veces antagónicos entre agentes conservacionistas, por un lado, y agentes económicos y sociales, por el otro, se plantea la posibilidad de realizar una explotación minera en zonas protegidas que impulse el desarrollo económico y social sin poner en riesgo la satisfacción de las necesidades de generaciones presentes y futuras. Dicha explotación debe ajustarse así al principio de desarrollo sostenible del derecho internacional ambiental y de la Unión.

La Directiva 92/43/CEE, del Consejo, de 21 de mayo de 1992³, relativa a la conservación de los hábitats naturales y de la fauna y flora silvestres (directiva hábitats), se desarrolló en un contexto internacional en el que la protección de la biodiversidad se consolidaba como un sector del derecho ambiental orientado hacia una utilización racional y sostenible de los recursos naturales. En esta se permite el desarrollo de actividades siempre y cuando no afecten la integridad de la Zona Especial de Conservación (ZEC), pero no se define este concepto.

A partir de un análisis encaminado a determinar la posibilidad de desarrollar proyectos de explotación de recursos naturales acordes con el ordenamiento ambiental internacional y comunitario, así como los propósitos que este persigue, se esclarece la posibilidad y las condiciones en que pueden desarrollarse dichos proyectos en las ZEC. Teniendo en cuenta que estos proyectos

3 DO L 206, de 22 de julio de 1992, p. 7. 
deben desarrollarse conforme al principio de desarrollo sostenible, se muestra, a través de los ejemplos de buenas prácticas de minería sostenible, la forma en que las actividades extractivas pueden generar un desarrollo social y económico, protegiendo a su vez el medio ambiente.

\section{DIVERSIDAD BIOLÓGICA Y SU PROTECCIÓN: EL CONCEPTO DE INTEGRIDAD DE LAS ÁREAS PROTEGIDAS}

\section{LA PROTECCIÓN DE LA DIVERSIDAD BIOLÓGICA EN EL DERECHO INTERNACIONAL}

En el derecho internacional, la protección de ciertas zonas en atención a su valor ecológico fue parte de los primeros tratados de derecho ambiental. La Convención Relativa a los Humedales de Importancia Internacional Especialmente como Hábitat de Aves Acuáticas (Convención de Ramsar, 1971) fue pionera en la creación de una Lista de Humedales de Importancia Internacional, en atención a parámetros ecológicos, botánicos, zoológicos, limnológicos o hidrológicos. La Convención procura principalmente un uso racional de los recursos naturales (arts. 2.6, 3.1, 6.2, 6.3), noción que, con el tiempo, sería progresivamente concretada. En 1987, con ocasión de la Tercera Conferencia de las Partes de la Convención (COP-Ramsar), el uso racional de los humedales fue definido como «su utilización sostenible para el beneficio de la humanidad de una forma compatible con el mantenimiento de las propiedades naturales del ecosistema» ${ }^{5}$. Esta definición fue posteriormente actualizada en 2005 por la COP-Ramsar, indicando que el «uso racional de los humedales es el mantenimiento del carácter ecológico, conseguido mediante la implementación de enfoques por ecosistemas ${ }^{6}$, dentro del contexto del desarrollo sostenible». Con ello - como señalan Beyerlin y Marauhn- la utilización racional termina equiparándose a una utilización sostenible 7 .

4 Instrumento de ratificación publicado en: BOE núm. 199, de 20 de agosto de 1982, pp. 22472-22474.

5 COP-Ramsar, Recommendation 3.3 (Regina, Canada, 27 May-5 June 1987).

6 COP-Ramsar, Decision V/6, anexo, A.1.

7 Ulrich BEYERLIN y Thilo MARAUHN, «Comparing this with the definition of sustainable use as included in Article $2 \mathrm{CDB}$, 'wise use' more or less equals 'sustainable use'»., International Environmental Law, Hart-CH Beck-Nomos, Oxford , 2011, p. 182. 
Más tarde, la Convención sobre la Conservación de las Especies Migratorias (Convención de Bonn, 1979) ${ }^{8}$ prevé la posibilidad de que los Estados partes suscriban acuerdos para la protección de especies migratorias cuyo estado de conservación sea desfavorable (art. IV, inc. 1). Posteriormente, en la lista de prioridades definida en la Cuarta Conferencia de las Partes de la Convención (COP-Bonn), se indicó que "[los] futuros acuerdos [...] deberán incluir disposiciones para la utilización sostenible de las especies cuando ello sea compatible con su conservación»", con el propósito de facilitar la suscripción de dichos acuerdos. Luego, dentro de los criterios para la evaluación de las propuestas de nuevos acuerdos, la COP-Bonn advirtió que "[las] propuestas deberán abordar todos los demás problemas importantes de viabilidad práctica para el lanzamiento y funcionamiento del Acuerdo (por ejemplo, estabilidad política o barreras diplomáticas para la cooperación) $»^{10}$. De esta forma, la Convención de Bonn busca, mediante una utilización sostenible, compatibilizar la conservación de las especies migratorias con las circunstancias del lugar en la que se aplica.

En 1979 fue aprobado también el Convenio relativo a la Conservación de la Vida Silvestre y el Medio Natural (Convenio de Berna, 1979) ${ }^{11}$. Allí se establecieron compromisos de adopción de medidas legislativas y reglamentarias apropiadas y necesarias para proteger los hábitats de las especies silvestres de la flora y de la fauna, y salvaguardar los hábitats naturales amenazados de desaparición (art. 4.1). Sin embargo, estas obligaciones quedan sujetas a excepciones «[...] en interés de la salud y de la seguridad pública, de la seguridad aérea o en atención a otros intereses públicos prioritarios» (art. 9, inc. 1). Con ello, se busca nuevamente una protección de las áreas y especies de especial interés ambiental acorde a otros intereses de cada lugar.

Así, a partir de dichos tratados, fue abordándose una protección jurídica de zonas de especial relevancia para la conservación de la diversidad biológica. Para ello, en todo caso, se flexibilizan las obligaciones de las partes en consonancia con sus circunstancias e intereses de importancia con el propósito, en definitiva, de conseguir una aplicación de los acuerdos alcanzados conforme a un criterio de sostenibilidad, es decir, que busquen la protección del medio ambiente sin dejar de lado el desarrollo económico y social.

8 Instrumento de ratificación publicado en: BOE núm. 259, de 29 de octubre de 1985, pp. 34071-34076.

9 COP-Bonn, Resolución 4.4 (Nairobi, 7-11 de junio de 1994), anexo, apdo. 16

10 COP-Bonn, Resolución 11.12 (Quito, 4-9 de noviembre de 2014), anexo, apdo. XI.

11 Instrumento de ratificación publicado en: BOE núm. 235, de 1 de octubre de 1986, pp. 33547-33555. 
La definición y la aceptación de la diversidad biológica o biodiversidad, como concepto omnicomprensivo de las relaciones entre los seres que forman parte del medio ambiente, ha sido objeto de mucha discusión, académica y científica $^{12}$. El punto de inflexión a nivel internacional, desde esta concepción limitada de biodiversidad en progresión hacia un concepto global con una perspectiva regulatoria de protección omnicomprensiva de la biodiversidad, lo marcó el Convenio de la Diversidad Biológica (CDB) suscrito en $1992^{13}$.

Dicho tratado amplió la protección de la diversidad teniendo en cuenta los ecosistemas y hábitats naturales en su conjunto y dando prioridad a la protección in situ de los mismos, siempre bajo el prisma de la utilización sostenible de los recursos de cada área que conservar (art. 8, CDB). El Convenio permite así que la gestión de un área protegida para la salvaguarda de la biodiversidad se compatibilice con el desarrollo de una actividad susceptible de generar un efecto perjudicial (art. 7, CDB) o efectos adversos importantes para la diversidad biológica (arts.10 y 14, CDB). En todo caso, dicha actividad debe ser identificada, categorizada y objeto de un procedimiento de evaluación de impacto ambiental que, cuanto menos, minimice los impactos. A esto se refiere la utilización sostenible de recursos naturales mencionada en el art. $10 \mathrm{CDB}^{14}$.

Esta orientación es, en definitiva, trasunto del principio de desarrollo sostenible ${ }^{15}$, que busca compatibilizar el desarrollo social y económico con

12 Irma NÚÑEZ, Edgar GONZÁLEZ-GAUDIANO y Ana BARAHONA, «La biodiversidad: historia y contexto de un concepto», Interciencia, vol. 28, núm. 7, 2003, pp. 387-393.

13 Instrumento de ratificación publicado en: BOE núm. 27, de 1 de febrero de 1994, pp. 3113-3125.

14 «Sustainable development proves to be a source from which subordinate norms may be derive one of which is 'sustainable use' in so far as it relates to natural resources. This precept calls upon states owning valuable natural resources on their territories, as well ass third states seeking access to these resources for exploitation, to use these resources in a sustainable manner, thereby ensuring their continuance. [...] Sustainable use is a special emanation of sustainable development. As such, it only gains normative quality if linked with a defined object of use. For example, the CDB [Convention on Biological Diversity] puts sustainable use together with components of biological diversity, such as flora and fauna, and other natural resources». Ulrich BEYERLIN y Thilo MARAUHN, op. cit., nota 5, p. 82.

15 Corte Internacional de Justicia, Sentencia de 25 de septiembre de 1997, Caso relativo al Proyecto Gab ikovo-Nagymaros, Hungría c. Eslovaquia, apdos. 140-141; y, Asamblea General de las Naciones Unidas, Resolución 60/1, aprobada el 16 de septiembre de 2005, 8a Sesión Plenaria, apdo. 48. 
la protección al medio ambiente. Dicho de otra forma, el CDB busca «que el desarrollo económico no suponga la destrucción de los recursos naturales o de las formas de vida tradicionales tan amenazadas de desaparición como la propia naturaleza» ${ }^{16}$. Para ello, como indican Sands y Peel, el CDB requiere la evaluación del impacto ambiental de los proyectos que puedan generar efectos adversos en las áreas protegidas, con el propósito de minimizar tales impactos ${ }^{17}$.

Por tanto, la protección de la biodiversidad no excluye el desarrollo de actividades que a priori puedan parecer perjudiciales para el medio ambiente y los objetivos del área protegida, máxime cuando de tal actividad pudiera depender el desarrollo social y económico de una comunidad. Debe, en todo caso, realizarse una evaluación de los impactos de la actividad, con el propósito de mitigar los efectos de dichas actividades.

\section{LA DIRECTIVA HÁBITATS}

La directiva hábitats, aprobada meses antes de la firma del CDB, fue innovadora por la definición de su ámbito de aplicación, los hábitats (art. 2.1), así como por la creación del principal instrumento de conservación de la biodiversidad en Europa: la denominada Red Natura 2000 (art. 3). También lo fue por la concreción de la obligación de los Estados miembros de establecer medidas y un procedimiento concreto de evaluación ambiental para todo aquel plan o proyecto que pudiera afectar de forma apreciable las ZEC (art. $6.3)^{18}$.

Es precisamente en este apdo. 3 del art. 6 donde aparece por primera vez el concepto de «integridad de la ZEC», como «integridad del lugar en cuestión", íntimamente unido al concepto "afección de forma apreciable de las ZEC». Sin embargo, en ningún otro apdo. del texto de la Directiva vuelve a hacerse referencia a tal concepto, ni mucho menos se delimita su definición o su contenido, dejando tal concepto abierto a interpretación.

En todo caso, el concepto «integridad del lugar» constituye el último filtro dentro de la evaluación ambiental a que todo plan o proyecto debe so-

16 Ana María BARRENA MEDINA, La protección de las especies silvestres: Especial tratamiento de la protección in situ (tesis doctoral), Universidad de Alicante, Alicante, 2012, p. 94.

17 Phillippe SANDS y Jacqueline PEEL, Principles of International Environmental Law, $3^{a}$ ed., Cambridge University Press, Cambridge, 2012, p. 457.

18 Julieta VALLS, Octavio INFANTE y Juan Carolos ATIENZA, Directrices para la evaluación ambiental de proyectos que puedan afectar a la Red Natura 2000, SEO/BirdLife, Madrid, 2014, p. 14. 
meterse, determinando la autorización o denegación de los mismos por las autoridades nacionales competentes. En esta evaluación deben conjugarse positivamente tres variables: tipo de afección del lugar, objetivos de conservación del mismo, e integridad de la $\mathrm{ZEC}^{19}$. Por tanto, el concepto «integridad de la ZEC» no puede quedar desligado de la clasificación propia de la ZEC en cuestión, la cual se delimita precisamente a partir de los objetivos de conservación de cada lugar. Dichos objetivos — como explica De Sadeleer - son los criterios que definen las características y condiciones ambientales de cada lugar ${ }^{20}$.

Es evidente además el componente técnico ${ }^{21}$ del concepto «integridad de la ZEC», por lo que el análisis del estado de conservación del lugar, tras el impacto del plan o proyecto y su incidencia en la integridad de la ZEC, habrá de atenerse a una metodología científica de comprobación, implementada siempre bajo los principios del derecho ambiental. Así lo entendió la Comisión Europea en su primer manual de interpretación de la Directiva, a los efectos de garantizar la coherencia de la Red Natura $2000^{22}$.

En la doctrina, por su parte, encontramos que los conceptos «integridad del lugar», «deterioro de hábitats», «alteración de especies» y las exigencias ecológicas de hábitats y especies del art. 6.1 se consideran equiparables como material de protección, conforme exige el art. 6 de la directiva hábitats ${ }^{23}$.

Se han avanzado ya dos problemas importantes que se dan en este momento. En primer lugar, el cuestionamiento o el afianzamiento del concepto «integridad en la ZEC» que se esté tratando, según los intereses de las partes intervinientes en el proceso de evaluación. En segundo lugar, la necesidad de desarrollar y elaborar unos criterios objetivos para evaluar los efectos de los proyectos extractivos realizados en las $\mathrm{ZEC}^{24}$.

19 Id. Además el preámbulo de la directiva hábitats, que considera la evaluación apropiada de «cualquier plan o programa que pueda afectar de manera significativa a los objetivos de conservación de un lugar que ha sido designado o que lo será en el futuro». Nicolas DE SADELEER, «Habitats Conservation y EC Law. From Nature Sanctuaries to Ecological Networks», The Yearbook of European Environmental Law, núm 5, Oxford University Press, 2005, p. 243.

21 Art. 12, apdos. a) y c) y art. 25.1 y 25.2; CDB, arts. 4.1 y 5.1, directiva hábitats.

22 Comisión Europea, Gestión de espacios Natura 2000. Disposiciones del art. 6 de la Directiva 92/43/CEE sobre hábitats, Luxemburgo, 2000, pp. 39 y 40.

23 Juan José PÉREZ PÉREZ, «Obligaciones de los Estados miembros en materia de gestión de los Espacios Natura 2000 derivadas de los apdos. 1 y 2 del art. 6 de la directiva hábitats", Revista Vasca de Administración Pública, núm. 99-100, (en memoria de Demetrio Loperena Rota), 2014, pp. 2426-2427.

24 Comisión Europea, Extracción mineral no energética y Natura 2000 (documento de orientación), Oficina de Publicaciones de la UE, Luxemburgo, 2011, p. 66. 
En esta tesitura, el Ministerio de Agricultura, Alimentación y Medio Ambiente del Gobierno de España promovió y financió un estudio sobre indicadores del estado de conservación de los hábitats de interés comunitario. Allí diseñó un sistema de indicadores que alerta sobre posibles disfunciones en el cumplimiento de los objetivos de la directiva hábitats ${ }^{25}$.

Si bien el concepto de integridad tiene su posición concreta dentro de los tres niveles establecidos para la evaluación ambiental ${ }^{26}$, la guía metodológica también lo relaciona directamente con los niveles superior e inferior derivados del art. 6.3 directiva hábitats. Conocer el estado de conservación es requisito primordial para su evaluación y mantenimiento (nivel 2). Mientras que la evaluación conjunta del grado de integridad individual del área protegida constituye uno de los factores importantes para identificar el grado de coherencia de la Red Natura (nivel 3). Para esto, se debe elaborar un mapa de todos los elementos que garantizan la preservación de la integridad del área protegida, con los que se puede identificar los elementos que han de formalizar y estimar el concepto de integridad ${ }^{27}$.

En resumen, el concepto «integridad de la ZEC» ha constituido un elemento novedoso en la regulación normativa de la protección de los ecosistemas europeos y en un ámbito internacional. Este concepto, complejo en su definición, resulta esencial y específico dentro del procedimiento de evaluación ambiental previsto en la directiva hábitats, al que debe someterse todo proyecto o plan susceptible de una afección importante en el área protegida. Para su delimitación habrá que tenerse en cuenta conceptos como el estado de conservación del hábitat o la especie protegida, los objetivos de conservación que delimitaron el área protegida, y la función ecológica del hábitat o coherencia con la Red Natura, siempre desde una óptica técnica y científica, que recoja in situ datos del estado de conservación inicial y de la problemática

25 Juan Carlos SIMÓN ZARZOSO, Ricardo GARCÍA MORAL, Gabriel DEL BARRIO ESCRIBANO, Alberto RUIZ MORENO, Sebastián MÁRQUEZ BARROSO y María E. SANJUÁN MARTÍNEZ, Diseño de una metodología para la aplicación de indicadores del estado de conservación de los tipos de hábitat de interés comunitario en España. Ministerio de Agricultura, Alimentación y Medio Ambiente, Madrid, 2013, pp. 5-25.

26 Nivel 1, Mantenimiento estado de conservación Hábitats de Interés Comunitario (HIC); Nivel 2, Mantenimiento integridad espacios RN 2000, y Nivel 3, Mantenimiento Coherencia RED.

27 SIMÓN ZARZOSO, GARCÍA MORAL, DEL BARRIO ESCRIBANO, RUIZ MORENO, MÁRQUEZ BARROSO, E. SANJUÁN MARTÍNEZ, op. cit., nota 23, p. 113. 
que la actividad a desarrollar puede generar en la integridad del hábitat o especie en cuestión.

Por otro lado, debe tenerse en cuenta que la protección de la biodiversidad en el ámbito de la Unión busca el desarrollo sostenible, en lo que se asemeja al derecho internacional ambiental en el sector de la biodiversidad. Como indica Alli Turrillas ${ }^{28}$, el fomento del desarrollo sostenible es el fin esencial de la estructura político-administrativa y competencial de la Unión Europea (UE), así como uno de los fines teleológicos de la protección del ambiente, arts. 3.3 y 3.5; y 21.2.d y 21.2.f del TUE.

Aparte, la directiva hábitats indica que las medidas adoptadas para la protección de la diversidad biológica deben tener en cuenta «las exigencias económicas, sociales y culturales, así como las particularidades regionales y locales» (art. 2.3). De esta forma, la Directiva se ajusta al ordenamiento internacional en el cual se busca la protección de la biodiversidad conforme a las circunstancias y los intereses de cada lugar.

Sin embargo, se observa que el texto del CDB no adopta el término «integridad de zonas o áreas que proteger», como un elemento para el seguimiento y mantenimiento de las mismas, a diferencia de lo que ocurre con la directiva hábitats. No fue hasta la Decisión VII/28, sobre áreas protegidas [arts. 8.a y 8.e] adoptada por la Conferencia de las Partes del CDB, cuando apareció el término al proponerse que la realización de los análisis de las lagunas del sistema de áreas protegidas nacional y regionalmente se basen, además de los criterios de identificación y seguimiento del anexo I del CDB, en otros tales como la integridad (objetivo 1.1, para 2010), al proponerse la rehabilitación y la restauración de la integridad ecológica de las áreas protegidas para la prevención y mitigación de los impactos negativos de graves amenazas a áreas protegidas (objetivo 1.5, para 2008); o para proporcionar un entorno institucional y socioeconómico favorable para el desarrollo de políticas en las áreas protegidas, identificando y estableciendo incentivos positivos que apoyen su integridad y mantenimiento, y contando siempre con la participación de las comunidades e interesados en la conservación (objetivo 3.1, para 2008).

Aparece, en definitiva, el concepto «integridad ecológica» en el desarrollo del para identificar qué se entiende por el término «integridad del área»o «integridad ecológica», y qué extensión tiene, coligiéndose, en definitiva, que el análisis del mismo habrá de ser casuístico.

28 Juan Cruz ALLI TURRILLAS, La protección de la biodiversidad: estudio jurídico de los sistemas para la salvaguarda de las especies naturales y sus ecosistemas, Dykinson, Madrid, 2016, pp. 79-81. 


\section{ANÁLISIS JURISPRUDENCIAL DEL CONCEPTO INTEGRIDAD DE LA ZEC}

El concepto de «integridad» constituye un elemento sustancial del procedimiento evaluador del plan o proyecto de la actividad que pretenda instaurarse y, por tanto, un elemento delimitador finalista de la autorización o denegación del mismo. En su definición entran en juego poderosos intereses contrapuestos que defiendan férreamente posturas extremas, en ocasiones antagónicas, en las que se confrontan los intereses y circunstancias de cada lugar, frente al sentido conservacionista, debiendo conciliarse estas posturas bajo el criterio de desarrollo sostenible.

El Tribunal de Justicia de la Unión Europea (TJUE) ha aportado unos criterios de interpretación sólidos del concepto de «integridad de la ZEC» en diversos pronunciamientos con los que, a priori, garantiza una interpretación bajo el principio de precaución del derecho ambiental:

a) los Estados no tienen discrecionalidad al momento de adoptar la decisión de autorización de un proyecto cuya evaluación justifique la pérdida o el riesgo grave de pérdida de un hábitat. Para ello, han de someterse a las obligaciones del art. 6.3 y 6.4 de la directiva hábitats siempre que existan valoraciones o conclusiones negativas de un proyecto sobre un hábitat ${ }^{29}$, de tal manera que solo podrán autorizar una actividad «si tienen la certeza de que no producirá efectos perjudiciales para la integridad de ese lugar ${ }^{30}$.

b) Se establece un binomio entre el compromiso de los objetivos de conservación de un hábitat y la consideración de la afectación de forma apreciable del mismo ${ }^{31}$. Así, la evaluación del impacto y de las repercusiones que pudiera tener un proyecto habrá de considerarse desde una evaluación previa del menoscabo de los objetivos de conservación del hábitat ${ }^{32}$, dentro de un proceso de evaluación adecuada entendido no como un mero acto administrativo formal, «sino que debe proporcionar un análisis en profundidad acorde con los objetivos de conservación establecidos para el lugar de que se trate»

29 Sentencia del TJUE, Comisión/Austria, C-209/02, EU:C:2004:61.

30 Sentencia del TJUE, Monsanto Agricultura Haly y otros, C-236/01, EU:C:2003:431, apdo. 59; y Sentencia del TJUE, Waddenvereniging and Vogels beschermingvereniging, C-127-02, EU:C:2004:482, apdos. 56 y 57.

31 Sentencia del TJUE, Waddenvereniging and Vogels beschermingvereniging, C-12702, EU:C:2004:482, apdo. 47.

32 Ibid., apdos. 45 y 46. 
debiendo estudiarse todas las repercusiones del plan o proyecto que incidan sobre los mismos ${ }^{33}$.

c) Se requiere una adecuada evaluación de las repercusiones del proyecto sobre los objetivos de la ZEC, la cual demuestre que con certeza no producirá efectos perjudiciales para la integridad de ese lugar. El margen de apreciación de las autoridades competentes queda así definido por la ausencia de «duda razonable, desde un punto de vista científico, sobre la inexistencia de tales efectos ${ }^{34}$.

d) Las características ecológicas de un lugar identificado por un Estado como Lugares de Interés Comunitario (LIC) ${ }^{35}$ supondrán también un límite para la autorización de planes o proyectos que pudieran alterar significativamente las características ecológicas de un LIC, siempre que tales intervenciones puedan conllevar el riesgo de reducir de forma significativa las características ecológicas de tales lugares $^{36}$.

e) Solamente cabe invocar razones imperiosas de interés público de primer orden (y de manera limitada) para permitir la autorización del plan o proyecto con incidencia en los objetivos de conservación del hábitat y, por tanto, con efectos perjudiciales para la integridad del mismo, siempre respetándose lo dispuesto en el apdo. 4 del art. 6 de la directiva hábitats ${ }^{37}$.

Con ocasión de la aplicación de la directiva hábitats, así como del art. 6 del Real Decreto 1997/1995, el Tribunal Supremo español (TS) ha tenido ocasión de pronunciarse en varias sentencias acerca del concepto de integridad. Asumiendo la jurisprudencia del TJUE, el TS ha considerado que la evaluación adecuada constituye un procedimiento taxativo y específico, con trámites de esencial trascendencia y sustantividad, sin posibilidad de aplica-

33 Sentencia del TJUE, Comisión/Holanda, C-441/03, EU:C:2005:233, apdos. 22 y 23.

34 Ibid., apdo. 25; Sentencia del TJUE, Waddenvereniging and Vogels beschermingvereniging, C-127-02, EU:C:2004:482, apdo. 67; y Sentencia del TJUE, Comisión/ Portugal, C-239/04, EU:C:2006:665; Sentencia del TJUE, Comisión/Irlanda, C-418/04, EU:C:2007-780.

35 Superficie, estructura y funciones, tamaño y densidad de población, grado de aislamiento de las poblaciones de especies, valor del lugar, etc.

36 Sentencia del TJUE, Bund Naturschutz in Bayern y otros, C-244/05, EU:C:2006:579; y Sentencia del TJUE, Comisión/España, C-308/08, EU:C:2010:281.

37 Sentencia del TJUE, Comisión/Holanda, C-441/03, EU:C:2005:233, apdo. 27. 
ción de interpretaciones flexibles ${ }^{1}$, al que todo plan o proyecto susceptible de afectar a lugares protegidos ha de someterse ${ }^{2}$. La evaluación es considerada además un mecanismo vital para acreditar la adecuación de los planes y proyectos a los objetivos de conservación del hábitat y la certeza de no causar perjuicio a la integridad del mismo ${ }^{3}$. La evaluación debe así desterrar todo tipo de incertidumbre sobre la inexistencia de efectos sobre la integridad del lugar, apoyándose siempre en importante substrato probatorio técnico, para evitar cualquier intento de justificación basado en el puro artificio dialéctico o retórico ${ }^{4}$.

\section{APLICACIÓN DEL ANÁLISIS AL SECTOR MINERO}

Los impactos de la minería sobre el medio ambiente son especialmente importantes, ya que se extienden al periodo posterior al cese de la actividad, lo que hace necesaria la restauración del lugar afectado. Como regla general, el proceso minero genera polvo y materiales contaminantes de roca sólida en el aire y las aguas 5 . Además, la actividad minera impacta severamente la biodiversidad en tres momentos importantes del proceso productivo minero, a saber: la sobreexplotación de recursos hídricos, la contaminación de las aguas por descargas de residuos y el impacto sobre el paisaje ${ }^{6}$.

Es pues un sector cuyos proyectos generarán una gran incidencia medioambiental, los cuales deberán ser sometidos al procedimiento de evaluación adecuada establecido en el art. 6 de la directiva hábitats, en los supuestos en los que se pretenda la instalación sobre áreas protegidas o aledañas y que pueda afectar de forma apreciable a las mismas.

1 TS (Sala de lo Contencioso Administrativo), Sentencia 2337/2010, de 7 de mayo.

2 TS (Sala de lo Contencioso Administrativo), Sentencias 1178/2004, de 24 de febrero 6493/2013, de 18 de diciembre. En sentido similar, Audiencia Nacional (Sala de lo Contencioso Administrativo), Sentencia 78/2011, de 17 de enero.

3 Audiencia Nacional (Sala de lo Contencioso Administrativo), Sentencia 5598/2006, de 11 de diciembre.

4 TS (Sala de lo Contencioso Administrativo), sentencias 3595/2016, de 13 de julio; 5005/2013, de 14 de octubre; 430/2011 de 14 de febrero, y 942/2013, de 5 de febrero.

5 Alianza Municipal de Derecho Ambiental (ELAW), Guía para evaluar EIAs de Proyectos Mineros, Eugene, EE. UU., 2010.

6 Eugenio FIGUEROA y Enrique CALFUCURA, "Principales actividades productivas y su relación con la biodiversidad», en Andrea SÁNCHEZ M. (ed.), Biodiversidad de Chile: patrimonio y desafios, CONAMA, 2006. 
En la práctica, se llegará al último filtro que exige la Directiva, la integridad del lugar, y se desatará el conflicto de intereses entre dos agentes. El promotor, que presentará una evaluación con identificación clara de la afección y, a su vez, una importante batería de medidas correctoras y alternativas viables; y el conservacionista, que evidenciará el lesivo perjuicio a la integridad del lugar para intentar obtener la denegación del proyecto. Nos encontramos en definitiva con un conflicto de intereses.

Antes de la directiva hábitats, el Pleno del Tribunal Constitucional español (TC) se pronunció sobre esta materia en su Sentencia 64/1982, de 4 de noviembre, en la cual aportó importantes conclusiones al respecto. Para el TC, ha de armonizarse la utilización racional de todos los recursos con la protección del medio ambiente, para garantizar el mejor desarrollo de la persona y la mejor calidad de vida conforme al art. 45 de la Constitución Española (CE). Por otro lado, indica que no cabe el establecimiento de una prohibición con carácter general de cualquier tipo de actividad extractiva, pues apartaría sin justificación alguna la atención que el poder público debe prestar a la modernización y el desarrollo de todos los sectores económicos (art. $130 \mathrm{CE}$ ) y vulneraría, por inconstitucional, el art. 128.1 CE, debiendo «armonizar la protección del medio ambiente con la explotación de los recursos económicos». Por último, es factible y constitucional que pueda declararse de forma expresa la prioridad de determinadas actividades extractivas sobre la protección del medio ambiente.

En resumen, y en palabras del propio TC, cuya doctrina ha sido reiterada en numerosa jurisprudencia del $\mathrm{TS}^{7}$, «la imposición de una carga adicional para la protección del medio ambiente no es en sí contraria a la Constitución ni al Estatuto, sí lo es la prohibición con carácter general de ciertas actividades extractivas de las secciones $\mathrm{C}$ y $\mathrm{D}$, que son las de mayor importancia económica, en una amplia serie de espacios».

Desde esta perspectiva inicial del Tribunal Constitucional, puede leerse también el contenido 3 del art. 6.3 de la directiva hábitats, entendiéndose que esta declaración expresa de priorización de determinadas actividades extractivas puede superar el requisito esencial de la afectación a la integridad de la ZEC, pero siempre justificado por razones imperiosas de interés público de primer orden, incluidas razones de índole social o económica.

La Ley 42/2007, de 13 de diciembre, del Patrimonio Natural y de la Biodiversidad $^{8}$, es más minuciosa en este tema. Los apdos. 5 y 6 de su art. 46

7 TS (Sala de lo Contencioso Administrativo), sentencias 699/1995, de 11 de febrero; 4756/2010, de 29 de septiembre; y 6423/2010, de 3 de noviembre.

8 BOE núm. 299, de 14 de diciembre de 2007, p.1. 
matizan con detalle cómo han de concurrir las razones imperiosas de interés público de primer orden y los motivos en los que han de basarse, tales como: la salud humana, seguridad pública, consecuencias positivas de primordial importancia para el medio ambiente u otras razones imperiosas de interés público de primer orden siempre previa consulta a la Comisión Europea.

Sin embargo, la priorización del sector minero frente a la protección del medio ambiente, basado en estas razones imperiosas de interés público de primer orden, no han de aducirse para evitar el procedimiento de evaluación, sino precisamente para justificar las medidas compensatorias que habrán de adoptarse al generarse un impacto sobre la integridad del lugar. Así lo expresó, con una estricta interpretación, el TJUE en la sentencia de 24 de junio de 2011 con ocasión de un recurso presentado, precisamente, contra España?

Sobre este aspecto también se ha posicionado expresamente la Comisión Europea, buscando la armonización entre la protección de la biodiversidad y el desarrollo socioeconómico. De un lado, la industria extractiva no energética (ENE) suministra muchas de las materias primas básicas para las actividades europeas de fabricación y construcción, existiendo casos en los que los planes y proyectos entran en conflicto con usos del suelo incompatibles, o con intereses sociales más amplios, incluidos los de conservación de la naturaleza. De otro lado, las directiva hábitats y la Directiva 2009/147/CE, relativa a la conservación de las aves silvestres ${ }^{10}$, constituyen la piedra angular de la política de la biodiversidad de la UE, teniendo como rasgo distintivo y fundamental la

9 «Procede recordar al Reino de España, que invoca la importancia de las actividades mineras para la economía local, que si bien tal consideración puede constituir una razón imperiosa de interés público de primer orden en el sentido del art. 6, apdo. 4, de la Directiva sobre los hábitats, esta disposición sólo resulta aplicable después de que se hayan analizado las repercusiones de un plan o de un proyecto de conformidad con el art. 6, apdo. 3, de dicha Directiva. En efecto, la determinación de estas repercusiones a la luz de los objetivos de conservación del lugar en cuestión constituye un requisito previo indispensable para la aplicación del citado art. 6, apdo. 4, ya que, a falta de esta información, no cabe apreciar si se cumplen los requisitos para aplicar esta excepción. En efecto, el examen de si concurren razones imperiosas de interés público de primer orden y de si existen alternativas menos perjudiciales requiere una ponderación con respecto a los perjuicios que el plan o el proyecto considerados causen al lugar. Además, con objeto de determinar la naturaleza de eventuales medidas compensatorias, los perjuicios causados a este lugar deben ser identificados con precisión». Sentencia del TJUE, 24 de junio de 2011, Comisión/España, asunto 404/09, EU:C:2011:768, apdo. 109.

10 DO L 20, de 26 de enero de 2003, pp. 7-25. 
creación de una red de lugares para salvaguardar las especies y tipos de hábitat más singulares y amenazados, la Red Natura 2000.

Partiendo de este encuadre, la Comisión considera que en la red Natura 2000 y a su alrededor no se establece ninguna exclusión automática de actividades ENE, si bien tales actividades habrán de atenerse a lo dispuesto en el art. 6 de la directiva hábitats, sometiéndose a una evaluación adecuada, para garantizar que no causan perjuicio a la integridad de los lugares Natura $2000^{11}$, debiéndose realizar el análisis que deba hacerse de cualquier proyecto minero, ha de realizarse "caso por caso" atendiendo a sus condicionantes e impactos ${ }^{12}$.

\section{BUENAS PRÁCTICAS PARA UNA MINERÍA SOSTENIBLE}

Al hablar de minería sostenible debemos tener en cuenta, ante todo, que esta actividad tiene por objeto recursos naturales no renovables, por lo que, como indica Dubińsky, la sostenibilidad de esta actividad debería comenzar por el consumo sostenible de minerales ${ }^{13}$. No obstante, no podemos obviar que el consumo y la extracción de minerales continúan en aumento, como muestran las estadísticas proporcionadas por el Sustainable Europe Research Institute ${ }^{14}$.

Ahora bien, conforme a lo anteriormente expuesto, el derecho internacional ha avanzado hacia una protección integral de la diversidad biológica que, a su vez, da prioridad a la adopción de medidas in situ, lo cual se concreta en el establecimiento de un sistema de áreas protegidas o áreas de especial conservación, cuya gestión debe permitir una explotación sostenible de los recursos naturales, que armonice el desarrollo social y económico con la protección ambiental.

11 Sentencia del TJUE, Azienda Agro-Zootecnica Franchin y Eolica de Altamura, C-210, EU:C:2051:502.

12 «[... la red Natura 2000 no es un sistema de estrictas reservas naturales de las que se excluya toda actividad humana. Por el contrario, las dos Directivas constituyen un marco legislativo común, aplicable en todos los países de la UE, que garantiza que las actividades humanas — entre otras las actividades ENE - se realicen de forma que no afecten negativamente a la integridad de los lugares Natura 2000». Comisión Europea, Guía sobre la realización de actividades extractivas no energéticas de conformidad con los requisitos de Natura 2000. Luxemburgo, 2011, pp. 7, 17, 19, 33 y 44.

13 Józef DUBIŃSKI, «Sustainable Development of Mining Mineral Resources», Journal of Sustainable Mining, vol. 12, núm. 1, 2013, pp. 1-6.

14 Sustainable Europe Research Institute, Overconpsumption? Our use of the world's natural resources, SERI, Global 2000, Friends of the Earth Europe, 2009, p. 9. 
La directiva hábitats supuso (y aún supone) el mayor avance en el propósito de la $\mathrm{CDB}$ de crear una sistema transfronterizo coherente de áreas de especial conservación para la protección del medio ambiente. Dicha protección conlleva, a su vez, la evaluación de cualquier plan o proyecto que pueda afectar la integridad de la zona protegida, de acuerdo con los objetivos de conservación que delimitaron el área protegida, su resistencia, su capacidad de evolucionar y la función ecológica del hábitat o coherencia con la red, lo cual deberá ser objeto de un análisis científico.

En el proceso de evaluación se enfrentan posiciones contrapuestas en las que se confrontan los intereses y circunstancias socioeconómicas de cada lugar, frente a posiciones conservacionistas, debiendo conciliarse estas posturas bajo el criterio de desarrollo sostenible. El desarrollo sostenible es un principio del derecho internacional ambiental, de acuerdo con el cual debe promoverse el desarrollo económico y social, protegiendo el medio ambiente en beneficio de las generaciones futuras ${ }^{15}$. En este orden de ideas, se reconoce que el desarrollo sostenible está formado por el desarrollo económico, el desarrollo social y la protección del medio ambiente, los cuales actúan como "pilares independientes que se refuerzan mutuamente» ${ }^{16}$.

En línea con lo anterior, el desarrollo de actividades de minería en los ZEC que favorezca el desarrollo sostenible debe promover el desarrollo social y económico, protegiendo a su vez el medio ambiente, lo que en cada caso se definirá de acuerdo con los objetivos de conservación confrontados con los intereses y condiciones concretas del lugar. A continuación, se muestran algunos modelos destacados de prácticas de minería sostenible que pueden ser tenidos en cuenta para la puesta en marcha de dichas actividades.

\section{LA PROTECCIÓN AMBIENTAL EN LA MINERÍA SOSTENIBLE}

Uno de las principales preocupaciones ambientales que genera la minería es ocasionada por el impacto que producen los residuos de esta actividad. Para responder a esta preocupación se han elaborado siete principios para determinar la sostenibilidad de la gestión de residuos de actividades mineras, de forma tal que esta afecte en la menor medida posible las condiciones de la economía, los ecosistemas y las comunidades en las que se desarrolla dicha actividad ${ }^{17}$.

15 SANDS, op. cit., nota 15, pp. 208 y 215.

16 Asamblea General de las Naciones Unidas, Resolución 60/1 de 24 de octubre de 2005, apdo. 48.

17 Daniel M. FRANKS, David V. BOGER, Claire M. CÔTE, David R. MULLIGAN, «Sustainable development principles for the disposal of mining and mineral processing wastes», Resources Policy, núm. 36, 2011, pp. 114-122. 
De acuerdo con estos principios:

a) los desechos de minería y de procesamiento de minerales deben ser manejados de manera que permanezcan físicamente, geográficamente, químicamente y radiológicamente estables.

b) La minería y los residuos de procesamiento mineral que interactúan con el medio ambiente deben ser inertes, es decir, equivalentes (en forma, concentración, ubicación, volumen, tiempo y valor) al material y productos químicos que ya están dentro del mismo ecosistema.

c) Los residuos de minería y de transformación de minerales que no sean inertes deben aislarse, hacerse lo más inertes posible, y en una forma compatible con la técnica de gestión de residuos adoptada y con la sensibilidad del ecosistema y del contexto social.

d) Los desechos minerales y de procesamiento de minerales deben ser confinados, es decir, geográficamente limitados, dejar una huella mínima en una ubicación de valores ecológicos y sociales aceptablemente bajos, y estar en forma física y química, dadas las condiciones locales, que limiten su interacción con el ambiente circundante.

e) Los desechos de minería y procesamiento de minerales deben ser manejados de acuerdo con las condiciones ambientales y sociales de cada lugar. La determinación de la aceptabilidad de la técnica de eliminación debe tener en cuenta las opiniones de las partes interesadas.

f) Los desechos minerales y de procesamiento de minerales deben ser manejados para minimizar la gestión posterior al cierre, los insumos (como el agua y la energía) y el volumen de desechos generados por volumen de un mineral extraído.

g) Las tecnologías de minería, procesamiento de minerales y gestión de residuos que ofrezcan un mejor desempeño ambiental y social, y ocupen un área menor, deben ser adoptadas preferentemente. En la medida de lo posible, deberán buscarse la reutilización de dese$\operatorname{chos}^{18}$.

18 Así, por ejemplo, de acuerdo con estos principios se aprecia que el sistema de tanques de relave, que es el más común en la actualidad, puede ser conveniente en los suelos áridos y semiáridos, en los que las precipitaciones son escasas, por lo que no se producen vertimientos contaminantes. Sin embargo, la estabilidad física es un punto débil de este sistema, debido ante todo a errores en la construcción, así como a movimientos telúricos. Por otro lado, con el sistema de relleno en pasta, la pulpa de relaves pasa 
Para adoptar una decisión sobre el sistema de gestión de recursos más adecuado para cada actividad, se ha elaborado además un sistema que tiene en cuenta el agua, la energía, los costes, los aspectos técnicos y los ambientales, integrando los componentes necesarios para una gestión sostenible ${ }^{19}$. Este proceso se divide en ocho pasos, a saber: a) caracterización geoquímica previa, para que los relaves no generen contaminación; b) identificación y análisis en laboratorio de las características y el comportamiento de los relaves; c) análisis de datos para determinar la relación entre el agua y el análisis y el consumo energético, identificando las opciones tecnológicas para la gestión de residuos; d) cálculo de los costos de cada opción a partir de los elementos principales; e) identificación y cálculo de los impactos ambientales de cada opción; f) identificación del marco regulatorio aplicable; g) inclusión de grupos de interés a través de un proceso de análisis de decisiones, $\mathrm{y}$ h) evaluación final a partir de los resultados de las fases previas ${ }^{20}$.

Dicho proceso ha permitido concluir en algunos casos que la opción de relleno en pasta tiene un menor coste, así como un menor impacto ambiental, pese a que suele concebirse como la opción más costosa en términos econó-

por procesos de separación sólido-líquido para incrementar el contenido de sólidos. Luego, esta se mezcla con cemento y escoria molida, para aumentar la resistencia del conglomerado. De esta forma, se consigue una estabilidad mayor, se reducen los vertidos y la toxicidad de los residuos, y se ocupa un área menor. Con ello se genera un impacto ambiental menor al de la anterior técnica, lo cual puede generar una inversión inicial más alta, pero unos costos de mantenimiento menores, por lo que puede considerarse la opción más sostenible. Por último, la técnica de disposición directa en ríos, lagos y mares puede alterar los parámetros físicos y químicos del lugar, incrementar los riesgos de inundación y generar daños en los ecosistemas acuáticos. Los desechos son además visibles, lo que genera molestias a la comunidad. Por ello, pese a sus costes bajos, se considera la opción menos sostenible. Ibid, pp. 117-119.

La fase de análisis de laboratorio desempeña un papel primordial, ya que a partir de una caracterización de los relaves puede identificarse la forma más efectiva de manejarlos. Las fases subsiguientes dependen de las propiedades físico-químicas de los relaves, realizándose en primer lugar un análisis de los datos provenientes de las pruebas de laboratorio, en los que se determina las posibles sinergias resultantes de la gestión del agua y la energía a través de un modelo jerárquico. A continuación, se examinan las tecnologías disponibles, de acuerdo con sus costes e impacto ambiental y, por último, se incluyen las expectativas y valores de los grupos de interés. Joni Safaat ADIANSYA, Michele ROSANO, Sue VINK y Greg KEIR, «A framework for a sustainable approach to mine tailings management: disposal strategies», Journal of Cleaner Production, núm. 108, 2015, pp. 1050-1062.

Ibid, pp. 1056-1058. 
micos. Así, por ejemplo, en el proyecto Osisko Hammond Reef (Canadá), la opción de relleno en pasta representó un ahorro del $40 \%$ en la construcción de la presa y del $19 \%$ en el bombeo de agua. En la mina Quebrada Honda (Perú) los costos operacionales de este sistema de gestión de residuos descendieron en un $19 \%$ respecto a los del sistema tradicional de relave. Y en la mina Century (Australia) la puesta en marcha del sistema de relleno en pasta generó un ahorro del $33 \%{ }^{21}$. Vemos así que la opción de gestión de residuos con mejor desempeño ambiental no es necesariamente la más costosa.

Otro de los aspectos de la minería que genera inquietudes se encuentra en la gestión del recurso hídrico, debido al alto consumo de agua que se presenta en la actividad extractiva y la contaminación que esta produce. Para realizar una gestión eficiente de los recursos hídricos subterráneos en la actividad minera se requiere, en primer lugar, tener conocimiento previo del estado en el que se encuentran las aguas superficiales, para determinar la probabilidad de que se presenten problemas. A partir de ello, debe - en segundo lugar - centrarse la atención en las áreas críticas o sobreexplotadas. En tercer lugar, debe hacerse mantenimiento a los pozos de agua y observar, a partir de ello, el cuidado que debe dársele a los cuerpos subterráneos. El aspecto normativo también es importante, requiriéndose una regulación clara en materia de aguas o, en su defecto, un sistema de incentivos y desincentivos, los cuales deben ser monitorizados, adoptándose las medidas necesarias ante su cumplimiento o incumplimiento ${ }^{22}$.

Pueden además adoptarse sistemas de «contabilidad de agua» que permitan ver con mayor claridad la forma en que la actividad minera da cumplimiento a los derechos humanos, incluido el derecho al agua. El agua derivada de la minería puede calificarse en tres categorías, como: a) aquella que requiere ser tratada levemente, para ser apta para consumo humano; b) aquella que debe ser tratada para remover todos los sólidos disueltos y otros componentes, para poder utilizarse para consumo humano, pudiendo, en todo caso, utilizarse sin tratamiento alguno para la agricultura, y c) las aguas hipersalinas, que no pueden ser utilizadas para la agricultura sin remover antes todos los sólidos en suspensión. A partir de esta clasificación pueden establecerse vínculos entre la gestión de aguas en la actividad minera y los derechos humanos ${ }^{23}$.

21 Ibid., pp. 1059-1060.

22 Sujay RAGHAVENDRA y Paresh Chandra DEKA, «Sustainable Developement and Management of Groundwater Resources in Mining Affected Areas: A Review», Procedia Earth and Planet Science, núm. 11, 2015, pp. 598-604.

23 Así, por ejemplo, cuando la actividad genere aguas de la primera categoría, la compañía minera estará contribuyendo al cumplimiento del derecho humano al acceso al 
Por otra parte, se ha comprobado que pueden disminuirse considerablemente los impactos ambientales de la actividad minera mediante prácticas de planificación estratégica, la implementación de tecnologías limpias, la conformación de agrupaciones de sostenibilidad y la extensión de la responsabilidad social $^{24}$.

Otra estrategia sobresaliente conjugó medidas de planificación estratégica, implementación de tecnologías limpias, conformación de agrupaciones de sostenibilidad y extensión de la responsabilidad social en Venezuela en la década de los noventa, con la colaboración de la empresa estatal $\mathrm{CVG}^{25}$. De

agua, mientras que cuando se estén ocasionando daños significativos sobre este tipo de aguas, podrá vulnerarse o ponerse en peligro este derecho. Un uso irresponsable de aguas de segunda categoría podría, por su parte, generar un menoscabo al derecho a la vivienda. Y, por último, cuando se empeoren las condiciones de aguas de tercera categoría, solo podría presentarse un incumplimiento de derechos humanos si el curso afectado tuviera un valor cultural. Ibid., p. 1159.

La compañía Placer Dome Inc., que a finales de los noventa era la segunda minera con mayor producción en Canadá, implementó con éxito modelos de gestión con estas características en las dieciséis operaciones que llevaba a cabo a nivel mundial. Para disminuir el impacto ambiental, esta compañía puso en marcha, en primer lugar, un programa de información ambiental con el que, a partir de reportes sobre las tendencias de contaminación generada por la actividad minera, se determinaban los cambios que debían realizarse en gestión de residuos, emisiones y vertidos. Aparte, adoptó el sistema de gestión ambiental ISO 14001, con el que, con base unas directrices sencillas, podía crearse un sistema empresarial coherente y predecible. Cabe destacar además que en África la empresa trabajó de la mano con el Gobierno para crear un nuevo marco normativo, hasta entonces existente, en materia ambiental. Adicionalmente, los técnicos y científicos de Placer Dome Inc. realizaban un seguimiento tecnológico continuo al avance de las técnicas de procesamiento de oro libres de cianuro. Para ello, la compañía estableció convenios con grupos de investigación y realizaron conferencias sobre formas de disminución de residuos. Gavin HILSON, Barbara MURCK, "Sustainable development in the mining industry: clarifying the corporate perspective», Resources Policy, núm. 26, 2000, pp. 227-238.

25 Con el propósito de poner fin a las técnicas móviles de dragado para la explotación de oro, las cuales generaban un enorme impacto ambiental en diversos puntos, se pusieron en marcha centros fijos de amalgamiento con mercurio operados por técnicos e ingenieros de la compañía. De esta forma, una gran compañía minera permitió, mediante procedimientos de transferencia de información y tecnología, que los mineros de pequeña escala implementaran técnicas menos contaminantes. Esta iniciativa fue replicada y optimizada por otras compañías, lo que permitió la operación de instalaciones de amalgamiento, como la de Carhuachi, en la que se recuperaba el $99 \%$ del mercurio utilizado. Marcello M. VEIGA y Christian BEINHOFF, «UNECA Centres: 
esta forma pueden resolverse conflictos entre mineros artesanales y de pequeña escala, y conformar organizaciones que fomentan el trabajo, liderazgo y conocimiento de las comunidades ${ }^{26}$.

La mina de oro McLaughlin en California, que operó entre 1985 y 2004, representa un modelo de gestión en el que la planificación estratégica, la implementación de tecnologías limpias y la concertación de acuerdos de sostenibilidad permitieron una explotación sostenible ejemplar. Parte de esta actividad se desarrolló en el condado de Napa, en el cual se produce un vino de denominación de origen de muy alto valor, por lo que es en la actualidad el suelo agrícola más costoso de Norteamérica. Es por ello percibida como una zona de alto valor ambiental, pudiendo presentarse un grave perjuicio a la industria vitivinícola en caso de que sucediera un accidente en la mina, así este no alcanzara directamente a los viñedos. Adicionalmente, la bahía de San Francisco, en la cual se desarrollo el proyecto, es la cuna e insignia de los movimientos ambientales en los Estados Unidos, por lo que cualquier actividad minera genera rechazo inmediato ${ }^{27}$.

a way to reduce mercury emissions from artisanal gold mining and provide badly needed training», Industry and Environment, vol. 20, núm. 4, 1997, pp. 49-51.

Janet M. EPPS, «The social agenda in mine development», Industry and Environment , núm. 20, vol. 4, 1997, pp. 32-35.

27 Pese a todo lo anterior, el proyecto McLaughlin fue aprobado con el apoyo de los grupos ambientalistas, y una buena parte del área en la cual se desarrollaron las actividades forma parte de una reserva natural en la actualidad. Para ello, se desarrolló un modelo de «cero vertidos», en el cual la tierra generada por las excavaciones fue utilizada para retener los relaves, así como para regenerar la vegetación adyacente. El diseño de la mina se realizó paralelamente al plan de gestión ambiental, no como un apéndice del primero. Aparte, la empresa minera dio muestras adicionales de responsabilidad ambiental, realizando un inventario de las plantas que se encontraban en un área de 30000 acres antes de la puesta en marcha del proyecto, reubicando una colonia de murciélagos que se encontraba en los túneles y descontaminando un área en la que anteriormente se encontraba una explotación de mercurio. Posteriormente, durante la fase de operación de la mina, se realizaron modificaciones en las labores para responder a los imprevistos ambientales que fueron surgiendo. En esta etapa se percibió que la posibilidad de que se presentaran drenajes ácidos era mayor a la esperada, ante lo cual se procedió a identificar los minerales que podían liberar tales drenajes, los cuales eran tratados y encapsulados separadamente en lugares de baja permeabilidad. Esto implicó un manejo flexible de las directrices ambientales para esta actividad minera, que en ocasiones iba más allá de lo exigido. Erica SCHOENBERGER, «Environmentally sustainable mining: The case of tailings storage facilities». Resources Policy, núm. 49, 2016, p. 122-123. 


\section{EL FACTOR ECONÓMICO EN LA MINERÍA SOSTENIBLE}

La minería puede traer consigo lo que se denomina la «enfermedad holandesa $»^{28}$, debido a que el ingreso de divisas procedente de la exportación de minerales puede producir una apreciación de la moneda nacional que disminuye con ello el nivel de competitividad de los sectores industrial y agropecuario $^{29}$. De esta forma, la expansión de la minería en lugar de contribuir al progreso económico produciría un efecto contrario. Los Gobiernos y la comunidad en la que se desenvuelve la actividad minera tienen actualmente conocimiento de esta posibilidad, ante lo cual se ha hecho necesario tomar medidas.

Adicionalmente, las comunidades locales son cada vez más exigentes sobre la forma en que deben distribuirse los beneficios derivados de la minería. La población ha ido concienciándose progresivamente sobre los impactos ambientales derivados de la actividad minera, con lo que podría producirse a nivel local un perjuicio mayor que el beneficio que trae consigo la minería en la comunidad en la que se desarrolla. A esto se suma el aumento de precios de los minerales generado por el incremento de la demanda proveniente de los países asiáticos, lo que - gracias a la extensa cobertura actual de las telecomunicaciones - es ampliamente conocido, impulsando las reclamaciones de las comunidades. Aparte, la minería se desarrolla en lugares cada vez más apartados, produciendo tensiones entre las empresas y las comunidades, las cuales buscan hacerse partícipes de los beneficios generados por la actividad que tiene lugar sus territorios ${ }^{30}$.

Todo lo anterior ha incentivado un cambio en el modelo de negocio practicado por las empresas mineras, ya que ignorarlo solo generaría problemas para el desarrollo de sus actividades. En respuesta a ello, se han creado

28 Conocida así por el masivo ingreso de capital derivado de la explotación del gas en la década de los sesenta, lo que trajo consigo una fuerte depreciación del florín. Al respecto: Teobaldo PINZÁS, «Interpretaciones de la relación entre el sector externo y la economía global», (Documento de Trabajo, 46), Serie Economía, 16, Lima, 1993, pp. 24-36.

29 Rodrigo UPRIMNY YEPES, y Nathalia SANDOVAL ROJAS, «Constitución, Industrias Extractivas y Territorios", Memorias del Encuentro Constitucional por la Tierra, Corte Constitucional de Colombia, Bogotá, 2015, págs. 228-229.

30 Ciaran O'FAIRCHEALLAIGH, «Community Development Agreements in the Mining Industry: an emerging global phenomenon», Community Development, vol. 44, núm. 2, 2013, pp. 222-238; y, David HUMPHREYS, «A Business Perspective on Community Relations in Mining», Resource Policy, vol. 26, núm. 3, 2000, pp. 127 131. 
mecanismos de beneficios compartidos (benefit-sharing) con los cuales se busca la distribución de beneficios monetarios y no monetarios generados en las regiones en las cuales se desarrollan las actividades ${ }^{31}$.

Dentro de los mecanismos de beneficios compartidos sobresale la creación de fondos para que los beneficios generados por la minería vayan más allá del ciclo de producción de las minas, proveyendo una fuente de financiación del desarrollo regional en el futuro. Según Fischer ${ }^{32}$, Drysdale ${ }^{33}$ y el Banco Mundial ${ }^{34}$, las mejores prácticas para la gestión de dichos fondos implican: a) $u$ único propósito por cada fondo; b) una visión estratégica clara sobre el papel del fondo en el ambiente local, un órgano administrativo compuesto por representantes de los diversos sectores afectados, un alto nivel de cofinanciación y colaboración entre los actores involucrados; c) la implementación de mecanismos de transparencia y rendición de cuentas sobre la forma en que se invierten los recursos del fondo, entre otros factores; d) evitar gastos excesivos que superen el nivel de asimilación por parte de la comunidad receptora, una estructura administrativa eficiente que maximice el desarrollo en las regiones, flexibilidad para adaptarse a las diversas y cambiantes condiciones operativas de las regiones, y e) un sistema tributario que no desincentive la inversión en la comunidad por parte de las empresas.

Para propiciar un desarrollo económico duradero para las comunidades en las cuales se desarrollan los proyectos de minería y se ajuste así a los intereses y las circunstancias del lugar en que se llevan a cabo, dichos fondos son destinados a inversión en capacitación, salud, infraestructura y diversificación económica. Esto - como se expone en el siguiente apartado- forma parte del desarrollo social y su puesta en marcha requiere acuerdos entre comunidades, empresas y Gobiernos, como los que se han conseguido en Chile, Estados Unidos (Alaska), Canadá y Australia. Debido al estrecho vínculo que, como se

31 Thu Thuy PHAM, Maria BROCKHAUS, Grace WONG, Le Ngoc DUNG, Januarti Sinarra TJAJADI, Lasee LOFT, Cecilia LUTTRELL, Samuel ASSEMBE MVONDO, Approaches to Benefit Sharing: A Preliminary Comparative Analysis of 13 REDD+ Countries (Working Paper), Center for International Forestry Research, Bogor (Indonesia), 2013, p. 108.

32 Carolyn FISCHER, International Experience with Benefit-sharing Instruments for Extractive Resources. Resources for the Future, Washington, DC, 2007.

33 Jennifer DRYSDALE, «Five principles for the management of natural resource revenue: The case of Timor-Leste's petroleum revenue», Journal of Energy and Natural Resources Law, vol. 26, núm. 1, 2008, pp. 151-174.

34 WORLD BANK, Mining Foundations, Trusts and Funds. A Sourcebook, Washington DC, 2010, 
observa, existe entre el desarrollo económico y social generado por la minería y las regiones, dichos ejemplos se exponen en el siguiente apartado.

\section{EL FACTOR SOCIAL EN LA MINERÍA SOSTENIBLE}

Teniendo en cuenta que «los seres humanos constituyen el centro de las preocupaciones relacionadas con el desarrollo sostenible» (principio 1, Declaración de Río, 1992), la minería sostenible debe buscar el desarrollo social de las comunidades del lugar en el que se desarrolla la actividad. Es por ello necesario el desarrollo de actividades encaminadas a conseguirlo, incorporando aspectos como la seguridad y estabilidad laboral, la salud de los empleados y de la población que reside en las proximidades a la unidad de producción minera, así como la diversificación y estabilidad económica de la región, entre otros aspectos que se muestran en este apartado.

Pero, ante todo, es necesario recalcar que, para conseguir el desarrollo social y económico, se requiere un trabajo colaborativo del Gobierno, las empresas mineras y las comunidades. El desarrollo social puede medirse a través de indicadores, como el Índice de Desarrollo Humano de Naciones Unidas en el que se conjugan la salud, educación y nivel de vida. Sin embargo, suelen presentarse discrepancias entre las comunidades, la industria y el Gobierno sobre el papel que corresponde a cada actor para conseguir el desarrollo social de una minería sostenible, así como sobre las actividades concretas en las que debe centrarse ${ }^{35}$.

En el ámbito de la responsabilidad social, mineras como Newmont y Goldfields han implementado programas de capacitación, microcrédito, infraestructura, vivienda, tecnificación del sector agrícola y becas escolares ${ }^{36}$. Goldfields, por su parte, implementa el Sustainable Community Empower-

35 Khadija BABIA, Hugo ASSELINB, Mostafa BENZAAZOUAC, «Stakeholders’ perceptions of sustainable mining in Morocco: A case study of the abandoned Kettara mine», The Extractive Industries and Society, num. 3, 2016, pp. 189-190. Marcellinus ESSAH, y Nathan ANDREWS, «Linking or de-linking sustainable mining practices and corporate social Responsibility? Insights from Ghana», Resources Policy, núm. 50, 2016, pp. 75-79.

36 Dentro de estos, cabe señalar a manera de ejemplo la Newmont Ahafo Development Fundation, a la cual se transfiere un dólar por cada onza de oro vendida y el $1 \%$ de las ganancias de la empresa. Gracias a ello, dicha fundación en 2015 contaba con trece millones de dólares, aproximadamente, para desarrollar su objetivo, que consiste en conseguir que la población afectada se encuentre en mejores condiciones a las que tenía antes del inicio de la actividad minera una vez haya cesado dicha actividad (dentro de 20 o 25 años). ESSAH y ANDREWS, op. cit., nota 71. 
ment and Economic Development Program (SEED), dedicado a mejorar las condiciones de vida de 30000 personas afectadas por las actividades que esta minera desarrolla en las dieciséis unidades de producción que desarrolla en Ghana $^{37}$.

Sin embargo, un significativo sector de la población se muestra aún insatisfecho con las medidas adoptadas. Para ellos, el principal problema social es el desempleo y, tras reconocer que no puede suministrarse empleo a toda la población, esperan que los beneficios se extiendan a toda la familia de los empleados. Aparte, recalcan que las regalías pagadas por las mineras no son invertidas en la satisfacción de las necesidades de la población afectada ${ }^{38}$.

Así pues, pese a que existen programas destinados a satisfacer necesidades sanitarias, educativas, así como de financiación e infraestructura, la población se encuentra insatisfecha en materia de desarrollo social. Ello - como observan las investigaciones de la Universidad de York (Canadá) — responde a la falta de criterios objetivos para definir en qué consiste el desarrollo social o a un acuerdo entre empresas y comunidades sobre lo que esto significa ${ }^{39}$.

\section{CONCLUSIONES}

En el ordenamiento internacional se acepta la utilización sostenible de los recursos naturales en los instrumentos relativos a la conservación de la biodiversidad conforme a los intereses y las circunstancias del lugar, advirtiendo, en todo caso, la posibilidad de actuaciones que puedan generar efectos adversos en los ecosistemas o en alguno de sus componentes, los cuales deben ser identificados para su eliminación o mitigación, reduciendo al mínimo los impactos ambientales adversos a largo plazo.

El concepto integridad ecológica ha constituido un elemento de significativa importancia en la regulación internacional y europea para la protección y conservación de hábitats, como factor esencial y específico dentro del procedimiento de evaluación ambiental al que debe someterse todo proyecto

37 Dentro de sus actividades, se han suministrado insumos para la agricultura, se han desarrollado programas de cultivo de palma de aceite, se provee alimentación a la población más vulnerable, como los niños y los enfermos de VIH o SIDA, y se han otorgado 390 becas. Ibid., pp. 79-82.

38 Las principales causas de desempleo en las localidades en las que se desarrollan las actividades mineras son la escasa capacitación, la negativa de los jóvenes a trabajar en actividades diferentes a la minería, como la agricultura, y la falta de capital para emprender actividades para las cuales fueron capacitados. Ibid., p. 83.

Ibid., pp. 82-85. 
o plan con claro impacto en el medio; factor que habrá de delimitarse con la valoración de otros conceptos como estado de conservación del hábitat, los objetivos de conservación del área y la función ecológica del hábitat. Este criterio debe conjugarse con otros elementos socioeconómicos que garanticen el desarrollo de los ámbitos donde se delimitan dichas áreas, conforme al principio de desarrollo sostenible del ordenamiento jurídico internacional y comunitario.

Es posible realizar una actividad minera que proteja el medio ambiente, para lo cual se han desarrollado con éxito mecanismos de gestión de residuos, gestión y contabilidad del recurso hídrico, prácticas de planificación estratégica y la implementación de tecnologías limpias.

La minería puede conseguir también un desarrollo socioeconómico de la comunidad en que se desarrolla. Para ello, debe conseguirse, en primer lugar, un acuerdo entre las empresas, las comunidades y, si es posible, el Gobierno, sobre las prioridades en materia de desarrollo social, y el papel que debe desempeñar cada uno de ellos para conseguirlo. Este acuerdo puede implementarse a través de mecanismos de beneficios compartidos, que han tenido éxito en varias latitudes.

Así, mediante una actividad minera que genere beneficios sociales y económicos, sin causar un menoscabo ambiental relevante, puede desarrollarse una minería sostenible en los ZEC. 